\title{
Desempleo e informalidad en América Latina: Definiendo políticas públicas para Venezuela
}

\author{
Osta Trestini, Karelys Mercedes*
}

\section{Resumen}

En este artículo se presenta una visión general del desempleo y la economía informal en América Latina, así como de su evolución en Argentina, Brasil, Chile, y Venezuela durante el período comprendido entre 1990 y 2004, incluyendo las políticas y programas relacionados con esta materia, a través de una investigación descriptiva de tipo documental. Finalmente, el aprovechamiento de los aprendizajes de la experiencia de estos países sirvió de base para la definición de políticas públicas para Venezuela dirigidas a proveer de capital humano y financiero a desempleados, trabajadores y empleadores de la economía informal que les permita tener acceso a oficios y a la creación de sus micro o pequeñas empresas con mayor productividad y valor agregado, contando con el respaldo de un marco legal adecuado y una institucionalidad capaz de dar respuesta eficaz, eficiente y oportuna a sus necesidades.

Palabras clave: Desempleo, economía informal, políticas públicas, América Latina.

\section{Unemployment and Informality in Latin America: Defining Public Policies for Venezuela}

\section{Abstract}

This article presents a general view of unemployment and informal economy in Latin America, and their evolution en Argentina, Brazil, Chile and Venezuela, between 1990 and 2004. The study includes the policies and programs related to this matter, using documentary, descriptive research. What was learned through the experiences of these countries served as a basis for defining public policies for Venezuela, directed to providing human and financial capital for the unemployed, workers and employers in the informal economy that would allow them to have access to jobs and the creation of their own micro or small businesses with greater productivity and added value, counting on the support of an adequate legal framework and an institutionality capable of giving an effective, efficient and opportune response to their needs.

Key words: Unemployment, informal economy, public policies, Latin America.

Recibido: 01-11-06. Aceptado: 10-06-07

Profesora Asociada de la Escuela de Ingeniería Industrial de la Universidad de Carabobo. Ingeniero Industrial (1992). Magíster en Administración de Empresas, Mención Mercadeo (2003). E-mail: kosta@uc.edu.ve, karelys35@yahoo.com 


\section{Introducción}

La década de los ochenta marcó un período de crisis originado por las políticas de sustitución de importaciones puestas en práctica en los distintos países de América Latina en los años precedentes, llevando a la aplicación de programas de ajustes estructurales cuyas consecuencias se han evidenciado durante los años noventas y el primer lustro del siglo XXI. En cualquier caso, las secuelas de la implementación de esas políticas están presentes (en mayor o menor grado) en toda la región, con su actual expresión en términos de desempleo e informalidad, como parte de las causas (o consecuencias) de la pobreza y la desigualdad; donde la institucionalidad y los actores que la representan (Estado, trabajadores, empresarios, universidades, comunidades) son elementos clave en la búsqueda de nuevos rumbos.

Es así como a través de este artículo, se busca presentar una visión general del desempleo y la economía informal en América Latina, así como de su evolución en el marco de las políticas de reformas estructurales aplicadas en Argentina, Brasil, Chile, y Venezuela durante el período comprendido entre 1990 y 2004, con el fin de identificar sus experiencias y fundamentar una propuesta en materia de institucionalidad y políticas públicas para Venezuela; donde la selección de tales países se justifica en virtud de su importancia económica y sus similitudes en cuanto a ubicación geográfica. El estudio se define como de tipo descriptivo de carácter fundamentalmente documental.

\section{Algunas precisiones}

Las primeras ideas que marcaron el inicio del debate sobre el sector informal se encuentran en el Informe de Kenya de la Oficina Internacional del Trabajo (OIT) (1972). A partir de ese momento, han sido elaborados diversos conceptos por parte de los organismos y entes nacionales e internacionales, los cuales han experimentado cambios en el transcurso del tiempo, afectando obviamente las cifras que pueden obtenerse de acuerdo con las distintas fuentes. En este sentido, esta investigación se basa en la definición operativa para las actividades informales en América Latina (y las cifras) de Panorama Laboral 2004 de la OIT, la cual:

"define a los ocupados en el sector informal como aquellas personas que son ocupadas y cuyo empleo principal se clasifica en una de las siguientes categorías: (1) trabajadores independientes (que incluye a los trabajadores familiares y a los trabajadores por cuenta propia, excepto los administrativos, profesionales y técnicos), (2) trabajadores en el servicio doméstico, y (3) ocupados en establecimientos que cuentan con hasta 5 trabajadores" (p. 86).

Sin embargo, ello no implica que se desecharán las múltiples aristas que se desprenden de los distintos enfoques teóricos en el tratamiento de este tema, los cuales, según lo plantea Rosenbluth (1994, citado por Veleda, 2001) se pueden resumir en el estructuralista, el neoliberaly el basado en nuevas formas de organización del trabajo

En cuanto a las estadísticas sobre población económicamente activa y, por 
ende, las de la tasa de desempleo, se presentan diferencias de acuerdo con las características de cada país; razón por la cual en este caso se optó por emplear la definición de la OIT (2004a), considerando como desempleados a:

"todas aquellas personas que tengan más de cierta edad especificada y que durante el período de referencia se hallen: "sin empleo", es decir, que no tengan un empleo asalariado o un empleo independiente; "corrientemente disponibles para trabajar", es decir, disponibles para trabajar en empleo asalariado o en empleo independiente durante el período de referencia; y, "en busca de empleo", es decir, que habían tomado medidas concretas para buscar un empleo asalariado o un empleo independiente en un período reciente especificado." (p. 86).

En cualquier caso, es importante recordar que el desempleo y la economía informal forman parte de un panorama global que aqueja a todos los países del mundo. Tal como lo señala la Oficina Internacional del Trabajo, para el año 2003 el desempleo total llegó a 185,9 millones de personas en busca de trabajo, pese al 3,2 por ciento de crecimiento del producto nacional bruto (PNB) en el mundo, reflejándose una mayor vulnerabilidad entre los jóvenes $(14,4 \%)$ y las mujeres $(6,4 \%)$. Por su parte, el tamaño de la economía informal creció en las regiones en desarrollo de poco aumento del PNB, destacando América Latina y el Caribe como la región más directamente afectada por la recesión económica mundial de 2001 (OIT, 2004b), sin olvidar su expresión en el trabajo infantil que en el año 2000 representó para esta región el $16 \%$ de los niños en edades comprendidas entre $5 \mathrm{y}$ 14 años (OIT, 2002).

\section{América Latina: la década perdida y los ajustes estructurales}

\subsection{Panorama General}

Para hablar de la situación de América Latina a partir de los años noventa, es necesario hacer referencia a las situaciones desencadenadas en la región durante la década anterior y el modelo de desarrollo que prevalecía en la mayoría de los países. El modelo de sustitución de importaciones implicó la protección de las empresas locales de la competencia internacional, subsidios, expansión del tamaño del Estado y de la deuda externa en procura del fortalecimiento de una industria nacional destinada a un mercado interno protegido.

Según el Banco Interamericano de Desarrollo (BID, 1994), las consecuencias se evidenciaron en los déficit de balanza de pagos ocasionados, en parte, por el continuado deterioro de los términos de intercambio de la región, el gasto excesivo del sector público, los intereses de la deuda externa sujeta a tipos de interés variable y la sobrevaluación de las tasas de cambio reales financiada a través de los incrementos del endeudamiento externo. Tal situación agudizó los problemas sociales existentes debido al estancamiento económico, contribuyendo a "la reducción del crecimiento de las oportunidades de empleo, la erosión de instituciones claves y el debilitamiento de los servicios públicos esenciales" como los sistemas de salud, infraestructura y, especial- 
mente, educación, originando una acumulación de necesidades sociales e institucionales (op. cit.) y el inicio de una etapa de ajustes en procura de lograr alcanzar el equilibrio macroeconómico.

La introducción de cambios como respuesta a los desajustes provocados por el modelo de sustitución de importaciones, orientados a incrementar la competitividad internacional y la búsqueda de una mayor participación de todos los estratos sociales en la distribución de los beneficios del crecimiento (op. cit.), implicó la aplicación de políticas de ajustes estructurales destinadas al restablecimiento del equilibrio de las cuentas nacionales a través de la reducción del déficit fiscal, eliminación de subsidios, reforma fiscal, liberación de tasas de interés y tasas de cambio, apertura a la inversión extranjera, privatización de las empresas en poder del Estado, resguardo a los derechos de propiedad, la desregulación en materia de nuevas inversiones, flujo de capitales, control de precios, barreras a las importaciones, barreras arancelarias y restricciones en cuanto a la reducción de personal en las empresas (Williamson, 2002), los cuales tuvieron diversas expresiones y matices en cada país.

Los resultados de los ajustes se expresan en distintos ámbitos. De acuerdo con el BID (1994), varios países de la región han logrado progresos en cuanto al restablecimiento del equilibrio macroeconómico y la introducción de cambios estructurales orientados a incrementar la competitividad internacional, lo cual ha incluido acciones para fortalecer un sector público de menores dimensiones, mayor disciplina fiscal, régimen de pagos y de comercio internacional más abierto, sistema de incentivos liberalizado que reduce la discrecionalidad en la administración de controles, licencias y subsidios, y al establecimiento de sistemas más equitativos y eficaces de prestación de servicios sociales.

En este contexto, el producto interno bruto (PIB) regional creció $3,7 \%$ en $1991,2,9 \%$ en 1992 y $3,3 \%$ en 1993 , siendo la recuperación del crecimiento especialmente notable en países como Argentina, Chile, Guyana y Panamá, cuyo PIB registró un aumento real promedio superior a 7 por ciento entre 1990 y 1993 (CEPAL, 1999 y BID, 1994) y logrando un progreso sustancial en cuanto a la reducción de la inflación desde niveles que, según datos de la Comisión Económica para América Latina y el Caribe (CEPAL), en la región superaba el $800 \%$ en 1993 a un 10\% en 1999 (op. cit.).

Las expectativas favorables creadas por el proceso de reforma estimularon la inversión privada, el auge en los mercados de valores y el aumento neto de las corrientes de capital, lo cual permitió la conversión del ahorro interno en formación de nuevo capital, incrementando la inversión interna bruta de la región un promedio de $8 \%$ entre 1991 y 1993 . Sin embargo, también aumentó la vulnerabilidad de sus economías a las fluctuaciones externas, como quedó demostrado con el estancamiento del PIB de la región durante las crisis de los años 1995 (crisis mexicana) y 1999 (crisis de los países asiáticos) (BID, op. cit.).

No obstante, los logros a nivel macroeconómico no han podido superar los problemas del desempleo y de economía informal, además de la pobreza y la exclusión, los cuales ya no afectan sólo a 
los más pobres sino también a clase media urbana (Quijano, 2004). Según la OIT (2004b), la tasa de desempleo en América Latina y el Caribe era del $6,9 \%$ en 1993, incrementándose al 8,0\% en el año 2003 y evidenciándose un máximo en el año 2002 del $9,0 \%$, mientras que al referir las cifras de desempleo urbano solamente a América Latina se reporta el paso de $8,0 \%$ en 1990 a un $11,7 \%$ en el año 2002 , para promediar durante los tres primeros trimestres del año 2004 un nivel del 10,8\%, siendo Venezuela la única economía de la región en la cual aumentó sensiblemente el desempleo entre el 2002 y el 2003, pasando del $15,9 \%$ al $18,0 \%$ (OIT, 2004a).

En cuanto a la economía informal, los datos de la OIT (2004a) muestran que en América Latina el porcentaje de empleo informal urbano respecto a la población ocupada creció del 42,8\% en 1990 a un $46,9 \%$ para el 2000 , disminuyendo levemente a $46,7 \%$ en el 2004 ; incremento que los distintos autores adjudican a aspectos como el aumento de la mano de obra por factores demográficos, ascenso del índice de actividad, particularmente de la mujer, migraciones significativas del entorno rural al urbano y reducción del empleo en la economía formal, que en el sector urbano pasó de $57,2 \%$ a $53,3 \%$ en el mismo período (OIT, 2002; Freije, 2002; Yánez, 2000; Veleda, 2001, entre otros).

En este marco, se ha conformado lo que Yánez (op. cit.) ha dado por llamar una "institucionalidad informal" como respuesta a la búsqueda del acceso a los bienes y servicios fuera del Estado y del mercado, aprovechando sus propias ventajas y características culturales. Tal si- tuación está dando lugar a distintos movimientos en los países de la región (Argentina, México, Brasil, Bolivia, Uruguay, Venezuela), donde la creciente masa de desempleados ha comenzado a orientarse más allá del reclamo de empleo, salarios y servicios públicos, organizándose en redes de producción autogestionaria y de autogobierno de tipo comunal (Quijano, 2004), incluso generando inestabilidad política en algunos de ellos.

Según el Banco Mundial (1998), los trabajadores del sector informal se caracterizan por poseer un bajo capital humano, involucrando en su mayoría a jóvenes y mujeres con poca experiencia laboral, y personas provenientes de las zonas rurales; mientras que entre los desempleados se identifican ciertas similitudes como un bajo capital humano (expresado, por ejemplo, en un nivel de instrucción primaria), jóvenes y mujeres sin experiencia, residentes en zonas urbanas y/o recientes inmigrantes, presentándose las mayores diferencias en cuanto a la menor disponibilidad de recursos y un nivel educativo inferior al del sector informal.

Sin embargo, autores como Freije (2002), Kantis et. al. (2003), Wormald y Salinas (2003) y Veleda (2001), entre otros, resaltan la heterogeneidad existente en el sector informal, donde el tradicional sector precario asociado con la pobreza y la explotación, se diferencia de otro más dinámico relacionado con la iniciativa empresarial y la utilización productiva del capital humano del empresario, generalmente apoyado en la red de relaciones familiares que le permiten el acceso a los recursos financieros y no financieros necesarios para llevar adelante sus actividades. 
Las políticas macroeconómicas también se evidencian en sus consecuencias en otras áreas, las relacionadas con el marco legal de cada país, afectando de una u otra manera el mercado laboral y la economía informal. Aunque no se pretende hacer un análisis exhaustivo de la situación específica en cada país latinoamericano, la autora trata de listar algunos aspectos presentes en materia laboral, fiscal, institucional y de derechos de propiedad, que han resultado inadecuados para dar respuesta oportuna y eficiente a las necesidades de recursos (financieros y no financieros) requeridos para la formalización de las iniciativas empresariales productivas en la región, ya sean individuales o colectivas, los cuales se señalan a continuación:

- Obstaculización de la creación y la operación de empresas formales, debido a las restricciones legales, requisitos y aranceles exigidos para el registro de empresas, que desembocan en pérdida de dinero y tiempo.

- Falta de ciertas regulaciones claves para la promoción de la empresarialidad, o la debilidad de las instituciones para lograr la aplicación de las leyes existentes.

- Elevados costos operativos relacionados con impuestos (nacionales, regionales y locales) y otras regulaciones que derivan de las relaciones laborales (impuestos sobre la nómina, indemnizaciones por despido, entre otros).

- En algunos países el salario mínimo no sólo afecta el empleo y la determinación de los salarios en el sector formal, sino también en el informal (Ma- loney y Núñez, citados por Freije, 2002).

- El aumento en la estabilidad laboral en el empleo formal y la deficiente aplicación de las regulaciones sobre el uso de la tierra en zonas urbanas se relacionan con un incremento en el empleo informal (Heckman y PagésSerra y Sassen-Koob, citados por Freije, 2002).

- La ausencia de títulos formales de propiedad impide que los propietarios realicen las inversiones necesarias y tengan acceso al financiamiento a través del sector financiero.

- Los obstáculos y las condiciones desfavorables (requisitos y elevadas tasas de interés) impiden el acceso al financiamiento de proyectos a través de la banca privada.

- El sector formal paga impuestos para financiar la provisión de servicios públicos y, en consecuencia, el sector informal tiene un acceso incompleto a tales servicios.

- El bajo nivel educativo de la población impide su adaptación a las exigencias de la incorporación de nuevas tecnologías en el sector formal y la ejecución de iniciativas empresariales más dinámicas en el sector informal (microempresarios).

En este sentido, la OIT (2004b) al plantear sus perspectivas refiere cómo los responsables en la definición de políticas deberían esforzarse para que el crecimiento de los países se plasme en un trabajo decente, creación de empleos en la economía formal, y "...no solamente... puestos de trabajo, menos productivos, en la informal" (p. 8). 
A partir de este panorama general, se consideró oportuno presentar una visión acerca del desempleo y la economía informal en América Latina durante el período comprendido entre 1990 y 2004 , específicamente en algunos de los países de la región, entre los cuales la autora seleccionó a Argentina, Brasil, Chile y Venezuela, así como estudiar las políticas y programas llevados adelante en ellos, fundamentalmente con el fin de extraer los aprendizajes que se desprenden de su aplicación en el contexto de los ajustes estructurales, de tal manera que puedan servir de base para la definición de políticas sobre estos aspectos en el caso venezolano.

\subsection{Argentina}

Desde principios de la década de 1990, con la puesta en marcha del programa de convertibilidad en 1991, Argentina comenzó a transitar un período durante el cual se alcanzaron varios de los equilibrios macroeconómicos básicos, el nivel de actividad agregada creció aceleradamente, se registraron avances en el grado de eficiencia productiva en varios sectores (Altimir y Beccaria, 1999) y una inflación controlada del 3,4\% en 1995 y hasta cifras promedio del $-1 \%$ entre 1999 y 2001 (OIT, 2004a).

Según Altimir y Beccaria (op. cit.), la evolución que mostró la desocupación entre 1991 y 1994 (de 5,9\% a 12,2\%) fue el resultado de dos efectos contrarios. Por un lado, la expansión económica favoreció la creación de puestos de trabajo fundamentalmente asociada al "boom" de consumo derivado de la estabilización, al crecimiento de las inversiones -ligadas a este mismo fenómeno y también a las privatizaciones- y de las exportaciones. Por el otro, la reestructuración productiva tendió a eliminar puestos de trabajo 0 a reducir la elasticidad del empleo a los crecimientos del producto, dado el incremento de la productividad del trabajo, que luego de algún tiempo de instauradas las reformas, se tradujo en el estancamiento del empleo.

La flexibilización de las regulaciones laborales constituyó uno de los elementos de las reformas estructurales de los noventas, argumentando la conveniencia de adecuar la legislación referente a las relaciones individuales y colectivas a fin de dotarla de mayor adaptabilidad y reducir los costos, especialmente, los costos laborales no salariales (aportes a la seguridad social, costos de despido y los derivados de ciertas regulaciones sobre duración de las jornadas, vacaciones, etc.), lo cual habría incentivado el uso de técnicas capital intensivas y disminuido la competitividad global de la economía. Además, se agregaba que la excesiva centralización de la negociación colectiva constituía un factor que limitó la flexibilidad salarial (en general) producto del papel del movimiento obrero, estrechamente relacionado con el peronismo.

Las diversas modificaciones en materia laboral apuntaron a reducir el costo laboral no salarial relacionado con las contribuciones patronales a la seguridad social y por concepto de despido (estableciendo diversas modalidades de contratos a tiempo determinado), accidente, aumentar la previsibilidad del costo laboral, flexibilizar la distribución del tiempo de trabajo y descentralizar la negociación de los contratos entre trabaja- 
dores y empresarios, las cuales estuvieron acompañadas por el establecimiento del seguro de desempleo en 1992, cuya cobertura a finales de 1997 apenas abarcaba al $6 \%$ de los desocupados (Altimir y Beccaria, 1999). Sin embargo, los cambios puestos en práctica, tuvieron poco impacto en los niveles de empleo.

El mercado de trabajo exhibió indicadores insatisfactorios, pasando de una tasa de desempleo urbano del $6,1 \%$ en 1991 al $17,3 \%$ en 1996, lo que representó el máximo de la década, superado solamente por el nivel alcanzado en el 2002 de un 19,7\%, y con una ocupación marcada por el comportamiento cíclico de la economía, reflejando los impactos de las crisis de los años 1995 y 2002 cuando llegó al $45,5 \%$ y $44,6 \%$ respectivamente. Otro elemento importante es la economía informal, la cual osciló entre un $52,0 \%$ y el $44,5 \%$ de esta población ocupada entre 1991 y 2002 (OIT, 2004a), evidenciando un aspecto de diferenciación con respecto a países como Brasil, México y Venezuela, pues los trabajadores desplazados del sector formal pasaron al desempleo abierto $y$, lejos de aumentar, el sector informal también experimentó una disminución.

En cuanto al comportamiento general de la economía, desde el año 1999 se produce una caída del PIB del $-3,4 \%$, la cual se atenuó un poco en el 2000 $(-0,8 \%)$, para luego continuar con un descenso más acentuado para el año 2001 $(-4,4 \%)$, que junto a la adopción de un conjunto de medidas económicas que incluyeron la restricción de la disponibilidad de los ahorros por parte de la población, desencadenaron la crisis política y social experimentada por Argentina entre los años 2001 y 2002, suscitando cambios de gobiernos, el levantamiento del programa de convertibilidad y de las restricciones al ahorro. Para el año 2002, la disminución alcanzada por el PIB fue de $-10,8 \%$, experimentando una recuperación de $8,5 \%$ en 2003 , la cual pudiera ser producto del efecto "rebote" de la economía.

De acuerdo con la OIT (2004a), la situación laboral en Argentina durante el 2004 presentó mejoría en cuanto a la reducción del desempleo abierto (a 14,6\%), debido a un aumento de la tasa de ocupación mayor al de la tasa de participación, el incremento de los salarios reales (en $57,8 \%$ ) y el aumento de la productividad, aunque con poco progreso con respecto a la informalidad.

\subsection{Brasil}

De acuerdo con Quijano (2004), Brasil constituye el único país que ha mantenido una importante estructura de producción industrial, con un capital financiero (propiedad de brasileños) ocupando una posición interna dominante, una destacada industria pesada que le permite producir e incorporar tecnología avanzada, además de contar con la presencia de empresas internacionales (como en el sector automotor). En palabras del autor, el país cuenta con la existencia de una fuerte burguesía brasilera, con intereses locales suficientemente importantes, un movimiento obrero extendido y con fuerza social, institucional y política, y con capas medias profesionales y tecnocráticas que no se han reducido.

De alguna manera Veleda (2001) ratifica esta visión cuando clasifica a Bra- 
sil entre los países con mayor nivel de desarrollo e influencia del sector financiero, donde los procesos de urbanización e industrialización empezaron antes que en otros de América Latina, favoreciendo de esta manera el surgimiento de movimientos sociales organizados que lograron alcanzar reivindicaciones diversas sobre los empresarios y el Estado, particularmente, en los segmentos urbanos donde se concentraba la mayor parte de la población y a quienes se dirigieron las políticas sociales. Es así como se evidencia una concentración de los beneficios del desarrollo económico en las áreas geográficas donde la producción ha mostrado mayor dinamismo, originando una gran disparidad regional y heterogeneidad en la composición social y los rasgos demográficos de los informales.

A mediados de los ochentas, Brasil sufrió una crisis que combinó elevadísimos niveles de inflación con un producto casi estancado, como el experimentado en América Latina. Es así como se despiertan serias reservas sobre la posibilidad de que el crecimiento económico fungiera como principal fuente de generación de nuevos empleos (Ramos, 2002), desde la perspectiva del estructuralismo, tanto por la percepción del agotamiento del proceso de sustitución de importaciones, en la periferia, como por la incapacidad de administrar la coyuntura por medio de los instrumentos macroeconómicos tradicionales en los países más desarrollados.

En consecuencia, en materia laboral se volcó la atención hacia las políticas dirigidas al mercado de trabajo, cuestionando en primera instancia su flexibilización, lo cual se refleja en los cambios marginales realizados en el conjunto le- yes agrupadas en la denominada Consolidación de las Leyes Laborales (CLT), y en el énfasis puesto tanto a las políticas activas destinadas a generar o mantener puestos de trabajo (formación profesional, intermediación y ayuda a las micro y pequeñas firmas), como a las políticas pasivas cuyo fin era reducir el desempleo mediante la disminución de la oferta de trabajo o la compensación financiera al trabajador desempleado por medio del seguro de desempleo (op. cit.).

En 1994, fue promulgada una ley que estableció el programa de seguro de desempleo, cuya finalidad era la de otorgar asistencia financiera al trabajador desempleado y auxiliarlo en su búsqueda de un nuevo empleo (centrada en la formación profesional e intermediación). Esta forma de intervención pública no difiere de los rasgos más amplios que tuvieron la casi totalidad de las políticas puestas en marcha en el área social: descentralización, cooperación con la sociedad civil, etc., las cuales constituyeron el marco institucional para el control y evaluación de las mismas (op. cit.).

Tratando de visualizar el contexto en el cual se ponen en marcha estas políticas, se puede resaltar como el desempleo urbano se ubicaba en un promedio de $4,9 \%$ entre 1990 y 1995, con una inflación del 66,0\%, una economía informal ubicada en el $46,5 \%$ de la población ocupada y un crecimiento del PIB de $4,2 \%$ para ese último año, según cifras de la OIT (2004a). En los años siguientes, la inflación disminuyó hasta alcanzar un promedio $8,5 \%$ entre 1996 y 2003 , aunque el desempleo se ha incrementado en el mismo período, pasando de $5,7 \%$ a $12,3 \%$ respectivamente. 
En cuanto al sector de la economía informal, destaca el aumento del sector de $40,6 \%$ en el año 1990 a un promedio de $45,7 \%$ entre 1995 y 2003 , el cual se debe fundamentalmente al aumento experimentado por el servicio doméstico (de 6,9\% en 1990 a $9,3 \%$ en 2003 ) y a los trabajadores en microempresas (de $13,5 \%$ a $14,3 \%$ ) (OIT, 2004a); sin embargo, habría que hacer estudios para determinar el impacto de la implementación de las políticas en el mercado laboral en este incremento de las iniciativas empresariales en Brasil destinadas a abarcar lo que Rosenbluth (citado por Veleda, 2001) denomina espacios económicos no cubiertos por la modernización o a la incapacidad del sector moderno para absorber en su totalidad la oferta de mano de obra, o a la alta inestabilidad ocupacional que generan algunas actividades promovidas por la modalidad de desarrollo.

\subsection{Chile}

En Chile, a diferencia de los otros países analizados, el proceso de ajuste estructural se inició a mediados de la década del setenta, involucrando una progresiva transformación de la economía desde las políticas derivadas del modelo de sustitución de importaciones, fuertemente protegido y dirigido por el Estado, hacia un régimen liderado por el capital privado y centrado en el desarrollo de una economía de mercado abierta al exterior y a la competencia derivada de la globalización. Este ajuste dirigido a mantener los equilibrios macroeconómicos y controlar la inflación, trajo consigo entre 1974 y 1984 la quiebra masiva de empresas, el aumento de la tasa de desocupación abierta (13,4\% promedio) y un crecimiento económico que apenas promedió un $2 \%$ anual (Wormald y Salinas, 2003).

Fue sólo a partir de 1985 cuando se consolidó el modelo de desarrollo exportador, iniciándose un período de progresiva reducción inflacionaria, de recuperación y crecimiento sostenido del producto y de la expansión del mercado de empleo hasta 1997; sin embargo, el país se expone a los ciclos mundiales, dada su mayor vulnerabilidad debido a la apertura. El año 1997 estuvo marcado por los efectos de la crisis asiática (op. cit.), la cual provocó un nuevo período de recesión cuando el PIB cayó de 6,7\% en ese año al -0,5\% en 1999, registrándose una recuperación promedio del $3,3 \%$ entre los años 2000 y 2003 (cifras OIT, 2004a).

Según datos de la OIT (op. cit.), el desempleo abierto (urbano) promedio del $9,1 \%$ entre 1998 y 2004 fue superior al registrado durante el primer lustro de la década de los noventas $(6,92 \%)$, con tasas de ocupación promedio del 48,8\%, manteniéndose la tendencia creciente de sus salarios reales (144,9 respecto a 1990), el control de la inflación (decreciente desde $6,1 \%$ en 1997 a $2,8 \%$ en el año 2003) y una economía informal que se conserva más o menos estable alrededor del $38 \%$ durante toda la década, lo cual coincide con lo planteado por Wormald y Salinas (aunque a través de fuentes distintas) sugiriendo que los desplazados de sus trabajos del sector formal en momentos de crisis, no se van inmediatamente al sector informal, sino que permanecen en el desempleo, en espera de recuperar su espacio en la formalidad.

Según Wormald y Salinas (op. cit.), este comportamiento de las tasas de de- 
sempleo y crecimiento del producto interno se ven complementados por la disminución de la tasa de crecimiento de la población económicamente activa (PEA) y el cambio en la potencialidad del aumento del producto para generar empleo. Según citan los autores, Infante señala que durante los noventas se evidenció una disminución de la oferta laboral en el mercado de trabajo chileno, atribuible fundamentalmente a la evolución demográfica del país en la que se reduce el crecimiento de la población en edad de trabajar y al retiro voluntario de la oferta laboral de aquellos que pierden la expectativa de encontrar ocupación con las características salariales o profesionales acordes con sus aspiraciones (principalmente mujeres), a lo cual los autores añaden el incremento de la cantidad de jóvenes que se dedican a estudiar (desde un $43 \%$ al inicio de la década a un $55 \%$ al final, según datos de las encuestas de caracterización socioeconómica de hogares Casen, varios años).

Por otra parte, la transformación de la estructura productiva (tercerización y subcontratación), con el incentivo de una economía que crece y expande la centralidad del mercado con un impulso al emprendimiento propio, se presenta como una consecuencia de la disminución del tamaño del Estado heredado del período anterior y de la mayor participación de los jóvenes en el proceso de educación, en concordancia con el crecimiento que en las últimas dos décadas ha tenido la oferta educacional, especialmente, en educación superior mediante la creación de universidades privadas, institutos profesionales y centros de formación técnica (Wormald y Salinas, 2003). Tal situación hace pensar en al menos una parte dinámica del sector informal, no necesariamente vinculado a la pobreza y a actividades de baja productividad.

Finalmente, se puede destacar que Chile presenta tasas de informalidad más bajas que el resto del continente $(38,8 \%$ en 2003) (OIT, 2004a), lo cual según Wormald y Salinas (op. cit.) puede ser el resultado de la expansión de los sistemas de seguridad social y del proceso de crecimiento económico que experimentó el país durante las últimas dos décadas. En el marco de ambos procesos, es posible hipotetizar que frente a una recesión (como la de estos últimos años) las expectativas de los desempleados no sea incorporarse a un trabajo informal por cuenta propia sino más bien mantenerse buscando trabajo para reincorporarse a un empleo, que pudiendo ser desprotegido, esté vinculado a empresas del sector formal.

\subsection{Venezuela}

Para tener una visión acerca de las características y evolución del desempleo y la economía informal en Venezuela desde los años noventa, es necesario tener presente las deficiencias en la diversificación de su economía y la vulnerabilidad derivada de los ciclos experimentados en el mercado petrolero internacional, lo cual ha marcado la inestabilidad del PIB (Banco Mundial, 1998) y la evolución de la estructura del empleo. Además, no hay que perder de vista los impactos generados por la crisis de la deuda externa durante la década de los ochenta y las consecuencias de la aplicación del conjunto de medidas de ajuste 
estructural que derivaron en la crisis social de 1989, que Quijano (2004) considera como el "punto de partida" de la expansión de la resistencia popular y la deslegitimación de ese modelo económico.

En términos generales, el comportamiento del PIB ha estado ligado a su componente petrolero, tal como lo indican diversos autores (Banco Mundial, 1998; Clemente, 2003; entre otros), a lo cual se suma una inflación que alcanzó casi el $100 \%$ en 1996 y que ha evolucionado hacia un $12,5 \%$ en el año 2001 , deteriorándose en los años 2002 y 2003 $(22,4 \%$ y $31,1 \%$ respectivamente) junto con la producción del país (-9,0\% y -9,3\% en esos años) (OIT, 2004a), debido a los conflictos fundamentalmente de orden político experimentados durante ese período.

En cuanto a la situación en el mercado laboral, entre los años 1991 y 1993 se evidencia una disminución en el nivel de desempleo con relación al reflejado en 1990 (11\%) llegando al 6,8\% en 1993, lo cual concuerda con la recuperación del PIB en ese período luego de la crisis de finales de los ochentas. para luego continuar su aumento hasta alcanzar en el 2003 el 18,0\% según las cifras de la OIT (2004a) o el 16,8\% según el Instituto Nacional de Estadística (INE, 2004), siendo estas últimas consecuencia de los problemas internos anteriormente referidos. Entre las características del empleo en el país resaltan la contribución del sector de la construcción a estos valores y el comportamiento estacional que experimenta la tasa de ocupación, poniendo en evidencia un marcado aumento durante el último trimestre del año.
En el año 1997, se llevan a cabo las modificaciones en la legislación laboral y el sistema de remuneraciones que permiten flexibilizar el mercado del trabajo formal y reducir la incertidumbre acerca de los costos laborales futuros a través de la eliminación del sistema de indexación de los pasivos laborales, medidas éstas previstas entre las políticas de ajustes estructurales.

En este proceso, la economía informal se convirtió en el mecanismo de escape para tratar de atenuar las consecuencias negativas derivadas de tal situación, pasando de un $38,6 \%$ de la población ocupada en 1990 a representar el $53,8 \%$ en 2003 , resaltando el incremento del 6\% reflejado entre los años 1995 y 2000 debido al aumento del sector de trabajadores por cuenta propia (de $28,1 \%$ a $34,5 \%$ ) que pudiera explicarse (al menos en buena parte) por la reducción del empleo tanto en el sector público (en un $3,8 \%$ ) como en el sector privado $(2,4 \%)$ (cifras OIT, 2004a).

Otro aspecto interesante observado entre los años 2000 y 2003, es el aumento de la participación del sector de las microempresas, pasando del $13,8 \%$ al $17,9 \%$, lo que representa un rasgo de iniciativa empresarial (voluntaria o forzada por las circunstancias), en especial entre la población masculina, que contrasta con la disminución sostenida del sector de la pequeña y mediana empresa (PYME), lo cual podría ser coincidente con los resultados obtenidos por Kantis et. al. (2003) acerca del papel de las PYMEs como incubadoras de nuevos emprendimientos y proveedoras de experiencia, tanto laboral como de gestión. 
En cuanto a la formación del capital humano, en el país existen instituciones y programas previstos en la legislación (algunas de larga data), públicas, privadas o mixtas (Instituto Nacional de Cooperación Educativa INCE, Fundametal, entre otros), destinadas a fortalecer el nivel educativo de la población y su capacitación para el trabajo, adicionalmente a la red que conforma el sistema educativo venezolano en todos sus niveles (preescolar, básica, diversificada, técnica y superior). No obstante, en algunas de ellas su labor ha estado enfocada a sectores que cuentan con empleos o a satisfacer necesidades específicas de las empresas, debilitando así su potencial para lograr la inserción de sectores importantes excluidos del mercado laboral.

Durante los últimos años, el gobierno nacional ha propiciado en el país iniciativas dirigidas a fortalecer la capacitación de los sectores más desfavorecidos de la población, a través del redireccionamiento de instituciones ya existentes (INCE) o la creación de distintos programas socioeducativos (misiones), acompañados de oportunidades de financiamiento con intermediación de entidades financieras estatales (Banco del Pueblo, Banco de Desarrollo de la Mujer, Banco de Desarrollo Bandes), y de la creación de instancias como el Instituto Nacional para la Pequeña y Mediana Industria, la Sociedad de Garantías Recíprocas, el Fondo de Garantía Recíproca para la Pequeña y Mediana Empresa, el Programa Comisión Europea-Venezuela, el Consejo Nacional de Promoción de Inversiones y empresas del sector público (Petróleos de Venezuela, S.A., PDVSA), todas ellas orientadas al proceso de creación y desa- rrollo de la microempresa, la pequeña y mediana empresa, así como de cooperativas, asociaciones comunitarias, enmarcándose en las nuevas tendencias de los organismos internacionales como CEPAL, BID, OIT, entre otros.

Sin embargo, en estas iniciativas se detectan debilidades tanto en su orientación e implementación como en su control, evidenciando la necesidad de apuntalar esfuerzos en procura de lograr que los beneficios derivados de su adecuada aplicación permitan la inclusión de la masa desempleada al mercado laboral.

Por otra parte, también es importante atacar los distintos ámbitos que permitan la "formalización" o "legalización" de las actividades realizadas en la economía informal a través de una institucionalidad donde los beneficios (capacitación, asesoría, financiamiento, titularidad de la tierra) sean superiores a los percibidos en su "institucionalidad informal" y a los costos asociados (impuestos nacionales y municipales, cotizaciones en seguro social, prestaciones sociales) a esta "formalización".

\section{Propuesta de políticas}

La situación del desempleo y la economía informal existente en América Latina, detallada a través de las experiencias de Argentina, Brasil, Chile y Venezuela, deja en evidencia algunos elementos acerca de la necesidad de alcanzar la consolidación de una institucionalidad (Estado, empresarios, representación de los trabajadores, universidades y centros de investigación, organizaciones no gubernamentales, asociaciones y cooperativas, sociedad civil, entre otros) capaz de 
conjugar esfuerzos conjuntos para dar respuesta eficaz, eficiente y oportuna, los cuales pueden ser sujetos de políticas públicas destinadas a mejorar la condición de los sectores de la población afectados, obviamente basadas en las distintas investigaciones y estudios referidos.

Finalmente, la autora propone las siguientes políticas públicas para Venezuela, sobre la base del fortalecimiento de la institucionalidad, el marco legal, el financiamiento y el capital humano, con miras a disminuir la incidencia del desempleo y la economía informal a través de la incorporación de esos sectores de la población al trabajo productivo en actividades de mayor productividad y valor agregado, en aras de lograr una mejor distribución de los ingresos y elevación de la calidad de vida de la población:

- Adecuar el sistema educativo con miras a fortalecer la formación de capital humano adaptado a las nuevas tecnologías, la necesidad de la población de contar con posibilidades reales de empleo y compenetrado con las circunstancias y potenciales de sus localidades y regiones.

- Conformar programas de formación orientados a lograr la capacitación de la población desempleada y ocupada en la economía informal, en especial de jóvenes y mujeres, para el desarrollo de habilidades y destrezas (a distintos niveles) adecuadas a las ventajas comparativas susceptibles de explotación en sus localidades. Éstos abarcan desde las áreas artesanales y técnicas relacionadas con los conocimientos y habilidades requeridos para un determinado oficio, pasando por la formación en el uso de nuevas tecnologías hasta las prácticas en gestión de micro y pequeñas empresas, elaboración de proyectos, obtención de financiamiento, liderazgo y desarrollo personal.

Las líneas generales del programa deben estar orientadas por el modelo de desarrollo, tomando en consideración las potencialidades de las regiones, y ser definidas a nivel nacional por un ente integrado por representantes gubernamentales, universidades, empresarios, sindicatos, organizaciones no gubernamentales, asociaciones de la economía informal y cooperativas; para luego operacionalizarlos a través de entes regionales y locales que permitan la descentralización y el acercamiento de esa formación a las realidades y potencialidades locales.

Todo ello con la finalidad de construir el capital humano y social necesario para su inserción en la economía formal, la generación de competencias que den acceso a la oportunidad de desarrollar actividades por cuenta propia, la creación y gestión de microempresas con expectativas reales de crecimiento o el impulso de proyectos colectivos (cooperativas y asociaciones) productivos con posibilidades de incursionar en mercados internacionales con el apoyo (asesoría, financiamiento) de instituciones sólidas, permitiendo la diversificación de la oferta exportable del país.

- Apoyar la creación y desarrollo de microempresas como medio para lograr la formalización de la actividad económica del sector informal, a través la agilización de los distintos trámites necesarios ante las instancias res- 
pectivas y la reducción de los costos relacionados con su registro.

- Proveer fuentes de financiamiento, capacitación y asesoría para la constitución de microempresas, así como los mecanismos de control que garanticen la utilización adecuada de los recursos, entre los cuales podría establecerse la ejecución de los mismos entre el ente financiero y los proveedores seleccionados por el empresario.

- Propiciar la constitución de asociaciones de representación de la economía informal, a través de la incorporación de las mismas como canales para la elaboración y tramitación de proyectos de carácter colectivo, tanto para su asesoramiento como para lograr su financiamiento.

- Proveer financiamiento para proyectos destinados a la construcción de infraestructura liviana para mercados, autogestión de mercados libres y periféricos, promovidos por las asociaciones de productores y campesinos y los trabajadores informales, con la finalidad de lograr su incorporación en el sector formal. Los recursos para dicho financiamiento pueden provenir del sector público (a través de los fondos existentes), organismos internacionales como el Banco Interamericano de Desarrollo, organizaciones no gubernamentales, aportes de cámaras empresariales, sindicatos y de los propios participantes, entre otros.

- Proveer de derechos de propiedad sobre las tierras (urbanas y rurales) y hacer cumplir los existentes, especialmente sobre la vivienda, ya que ella puede constituirse en un activo que le permita a la población tener acceso a fuentes de financiamiento, especialmente, cuando se trata de microempresas familiares.

- Poner en práctica los principios establecidos en las leyes vigentes en el país en cuanto a la reforma del sector público, para lograr el fortalecimiento de las instituciones, especialmente el sistema de justicia, y proveer a los gobiernos con instrumentos modernos dirigidos a garantizar el cumplimiento de las leyes y el ejercicio eficiente de sus funciones en apoyo al proceso de desarroIlo, sustentado en la incorporación de los avances tecnológicos en la búsqueda de la agilización de todas las tramitaciones necesarias ante las distintas instancias.

- En materia de legislación laboral, es necesario reflexionar y evaluar los beneficios "reales" de la flexibilización, pues existen experiencias a favor (caso chileno) y en contra (Brasil) acerca de los mismos.

- Concluir los trámites pertinentes para proveer de una legislación en materia de seguridad social (vejez, salud, accidentes de trabajo y desempleo), en la cual se prevea la flexibilización necesaria para lograr la incorporación de los trabajadores de la economía informal a los beneficios que de ella se deriven. Adicionalmente, es indispensable lograr un sistema de seguridad social capaz de dar respuesta oportuna y eficiente a las necesidades de la población, en especial, en lo concerniente a las prestaciones en salud y vejez. 


\section{Consideraciones Finales}

Los programas de ajustes estructurales aplicados en América Latina han tenido logros en el restablecimiento de los equilibrios macroeconómicos; sin embargo, no han sido capaces de superar las secuelas que en cuanto al desempleo, la economía informal, la pobreza y la distribución de los ingresos se refiere. Las experiencias de Argentina, Chile, Brasil y Venezuela, arrojaron distintos matices, circunstancias y resultados sobre el abordaje de las políticas dirigidas a alcanzar el desarrollo, donde cada una evidencia sus propios avances y debilidades.

En este sentido, la posibilidad de aprovechar los aprendizajes de cada experiencia para definir políticas públicas dirigidas a proveer de capital humano y financiero a desempleados, trabajadores y empleadores de la economía informal, que les permita tener acceso a oficios y a la creación de sus micro o pequeñas empresas con mayor productividad y valor agregado, contando con el respaldo de un marco legal adecuado y una institucionalidad (Estado, empresarios, representación de los trabajadores, universidades y centros de investigación, organizaciones no gubernamentales, asociaciones y cooperativas, sociedad civil y otros) capaz de conjugar esfuerzos para darles respuesta eficaz, eficiente y oportuna, es una opción válida para disminuir el impacto de estos fenómenos y reducir las desigualdades en la distribución del ingreso, aminorando su impacto en las condiciones de pobreza en Venezuela.

\section{Referencias Bibliográficas}

Altimir, Oscar y Luis Beccaria (1999). El mercado de trabajo bajo el nuevo régimen económico en Argentina. Serie Reformas Económicas, № 28. Comisión Económica para América Latina y el Caribe (CEPAL).

Banco Interamericano de Desarrollo (BID) (1994). Perspectivas de crecimiento y desarrollo social en América Latina. Consultado el 05/12/04. Disponible en: http://www.aguabolivia. org/analisisX/INTERNACIONAL/BID/ CrecimientoAL.htm

Banco Mundial (BM) (1998). Stylized facts and the characteristics of the labor supply in Venezuela. What can be done to improve the outcome? Report No. 17901 -VE.

Comisión Económica para América Latina y el Caribe (CEPAL) (1999). Balance preliminar de las economías de América Latina y el Caribe. Consultado el 10/12/2004. Disponible en: http:// www.eclac.cl/cgi-bin/getProd.asp? $\mathrm{xml}=/$ publicaciones/xml/4/4524/P4 524.xml\&xsl=/de/tpl/p9f.xsl\&base=/ tpl/top-bottom.xslt

Clemente, Lino (2003). Crecimiento económico y productividad en Venezuela, 19502000. Revista BCV, Volumen XVIII, № 1. Enero-junio, pp. 13-40. Banco Central de Venezuela: Caracas.

Freije, Samuel (2002). El Empleo Informal en América Latina y el Caribe: Causas, consecuencias y recomendaciones de política. Banco Interamericano de Desarrollo. Consultado el 30/11/2004. Disponible en: http://idbdocs.iadb.org/wsdocs/getdocument. aspx?docnum $=354774$

Instituto Nacional de Estadística (INE) (2004). Informe Semestral de Fuerza Laboral. 2을 Semestre 2003. Consultado el 
12/12/2004. Disponible en: http:/ /ww w.ine.org.ve

International Labor Organization (ILO) (1972). Employment, incomes and equality: a strategy for increasing productive employment in Kenya. International Labour Organization: Geneva.

Kantis, Hugo, Masahiko Ishida y Masahiko Komori (2002). Empresarialidad en economías emergentes: Creación y desarrollo de nuevas empresas en América Latina y el Este de Asia. Banco Interamericano de Desarrollo. Departamento de Desarrollo Sostenible. Subdepartamento de Empresa Privada y Mercados Financieros. División de Micro, Pequeña y Mediana Empresa.

Oficina Internacional del Trabajo (OIT) (2004a). Panorama Laboral 2004. América Latina y el Caribe. Consultado el 03/01/05. Disponible en: www.oit.org.pe/portal/documentos/ panorama_laboral2004.pdf

Oficina Internacional del Trabajo (OIT) (2004b). Tendencias mundiales del empleo. OIT: Ginebra.

Oficina Internacional del Trabajo (OIT) (2002). Informe VI: El trabajo decente y la economía informal. Conferencia Internacional del Trabajo. 90ª reunión. OIT: Ginebra.

Quijano, Aníbal (2004). El laberinto de América Latina: ¿Hay otras salidas? Revista
Venezolana de Economía y Ciencias Sociales, año/vol. 10, enero-abril, № 001, pp. 75-97. Universidad Central de Venezuela: Caracas.

Ramos, Carlos (2002). Las políticas del mercado de trabajo y su evaluación en Brasil. Serie Macroeconomía del Desarrollo, № 16. CEPAL: Chile.

Veleda, Susana (2001). Trabajo informal en América Latina: el comercio callejero. Universidad de Barcelona. Revista Bibliográfica de Geografía y Ciencias Sociales, № 317. Consultado el 14/01/05. Disponible en: http://www. ub.es/geocrit/b3w-317.htm

Williamson, John (2002). Chapter 2 from Latin American Adjustment: How Much Has Happened? Institute for International Economics.

Wormald, Guillermo y Viviana Salinas (2003). Informalidad en Chile durante la década de los años 90s. Population Research Center, University of Texas Austin. Consultado el 05/03/05. Disponible en: http://www.prc.utexas. edu/urbancenter/documents/report/ Latin_American_Urbanization/Chile \%20C\%20Informalidad.doc

Yánez, César (2000). América Latina en los noventa: los déficits del crecimiento. Consultado el 14/01/05. Disponible en: http://www.americaeconomica.net/reportajes2/ yanez.htm 